\title{
Targeted translational profiling
}

\author{
Tagging ribosomes in a cell type-specific \\ way allows the isolation of mRNAs that are \\ being translated in these cells.
}

\author{
"If the human brain were so simple \\ that we could understand it, \\ we would be so simple \\ that we couldn't." \\ -Emerson Pugh (in The Biological Origin \\ of Human Values)
}

We have come a long way since Emerson Pugh's assessment of brain research a few decades ago, and yet, the most complex organ in the body is still not an open book.

Nathaniel Heintz, a neuroscientist at New York's Rockefeller University, looks back at over a decade of trying to characterize the different cell types in the intact brain. Five years ago he, together with his colleague Mary Hatten, established the gene expression nervous system atlas (GENSAT), a large database of brain images derived from a plethora of transgenic mice, each expressing GFP in a particular cell type. These pictures are a valuable resource, but they still leave something to be desired. Heintz says: "We were frustrated with not knowing, at the molecular level, how cells might change during development, in disease or in response to different experiences."

Initially the Heintz team attempted expression profiling of cells by using fluorescenceassisted cell sorting. Although they had good success when sorting GFP-labeled cells from embryos, the mature brain proved much more refractory to this approach. It was impossible to dissociate specific cells from the intricately connected brain tissue without introducing stress responses that distort the molecular profile. The way to go was to directly isolate the mRNAs that were being translated.

Heintz reasoned that because all messages that are being translated are resident on ribosomes, a genetic handle on a ribosomal protein would allow isolation of the mRNA without having to isolate the cells. Joining forces with
Paul Greengard, another neuroscientist at Rockefeller University, they developed the translating ribosome affinity purification, or TRAP, methodology.

The concept, beautiful in its simplicity, took several years of tireless effort on the part of a team led by Myriam Heiman in the Greengard lab, to come to fruition.

Their first step was to identify a ribosomal protein that could be tagged with GFP. Next the scientists generated transgenic mice from bacterial artificial chromosomes in which this protein was under the control of a cell typespecific promoter. GFP-tagged ribosomes would then be purified via antibodies to GFP, the attached mRNA would be isolated, reverse transcribed and characterized. The key to this procedure is, of course, speed and specificity. The extraction of the ribosomes has to be fast, and dissociation of mRNA or, worse, reassociation of nonspecific mRNA to the ribosome must be avoided.

The essential resources are the transgenic mice expressing the GFP-tagged ribosomal protein. Heintz stresses the importance of ensuring that these mouse lines are wellcharacterized and shares his team's experiences: "We generated multiple lines for each cell type and did very detailed anatomical studies to confirm that the trap construct is expressed in precisely the right cell population at high enough levels."

In the first report presenting the TRAP method, recently published in Cell, the researchers analyzed the mRNA of four neuronal cell types on DNA microarrays. The researchers stimulated mice with cocaine and monitored translational changes in the neuronal cells. Although the arrays are sensitive enough to detect minor changes in expression, they are not quantitative; Heintz and Greengard are planning to perform nextgeneration sequencing to get a more quantitative picture of the changes. In addition, sequence data will yield information about alternative splice products and provide a more detailed picture of the molecular profile.

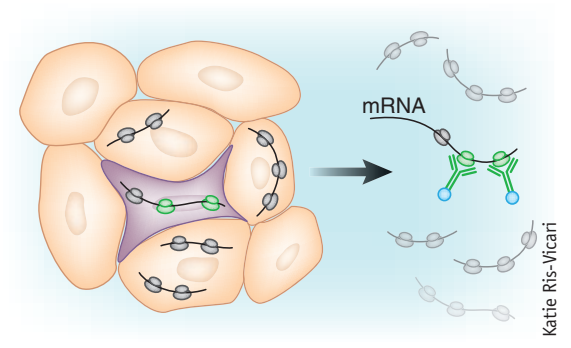

From cell-specifically tagged ribosomes to isolated mRNAs.

Heintz's goal for TRAP goes far beyond characterizing isolated cell types. He is interested in comparative analysis that will allow the clustering of the thousands of transcripts expressed in one cell type into smaller groups that are shared between certain cell types. Heintz is convinced that only assessing which cell types share which expression modules will give us functional insight.

Translational profiling may also lead to a new understanding of what constitutes a cell type. Heintz would like to see a cell type defined as a class of cells that share the same molecular 'ground state', that is, they share the same fundamental properties, although their responses to external stimuli may vary.

Heintz does not see the impact of TRAP as limited to studying mouse brain but envisions the method as a tool to study cell heterogeneity across different species. Once translational profiles have helped define cell types, antibodies to these cell types can be created and tested in other species. "This would allow us," Heintz says, "to address the century old question of what happened to specific cell types during brain evolution and how cell types differ in humans and lower mammals."

Nicole Rusk

\section{RESEARCH PAPERS}

Heiman, M. et al. A translational profiling approach for the molecular characterization of CNS cell types. Cell 135, 738-748 (2008). 